\title{
Atomic Defect Structure and Electron Transport Property of High-Speed Resistivity-Changing Nanoceramics
}

\author{
Masayuki FUJIMOTO \\ Graduate School of Science and Technology, Shizuoka University, 3-5-1, Jyouhoku, Hamamatsu, Shizuoka 432-8561
}

\begin{abstract}
Electron-transport mechanisms of oxide ceramics exhibiting high-speed voltage-pulse-induced resistivity changes were characterized with regard to nanostructure and defect formation. La-doped $\mathrm{SrTiO}_{3}$ single crystals with Ag top electrodes and Pt bottom electrodes exhibited bipolar resistive switching but retained the low-resistivity state for only 3 or $4 \mathrm{~h}$ because of unstable deep-level trap states at the metal-semiconductor interface. Crystalline $\left(\mathrm{Pr}_{0.7} \mathrm{Ca}_{0.3}\right) \mathrm{MnO}_{3}$ thin films sandwiched by $\mathrm{Pt}$ electrodes showed metallic conductivity and consequently never showed electric-pulse-induced resistivity changes, but insulative amorphous $\left(\operatorname{Pr}_{0.7} \mathrm{Ca}_{0.3}\right) \mathrm{MnO}_{3}$ thin films showed monopolar resistivity switching that suggested the formation of nanoscale filament paths with nanodomain switches. The $\mathrm{TiO}_{2}$ anatase nanolayer formed on a TiN thin film exhibited high-speed electric-pulse-induced bipolar resistivity changes thought to be due to a Mott transition caused by $\mathrm{o}^{2-}$ migration and the formation and annihilation of $V_{0} \ddot{\text { in }}$ 2.5-nm-thick anatase layer.

[Received July 19, 2007]
\end{abstract}

Key-words : Resistivity change, Strontium titanate, Manganite perovskite, Titanium oxides, Mott transition, Electron energy loss spectroscopy

\section{Introduction}

$\mathbf{I}_{\mathrm{th}}^{\mathrm{N}}$ MPURITIES in semiconductors are widely known to cause the formation of point defects such as donor and acceptor states and of microstructures such as planar faults and precipitates. Microelectronics has essentially been based on silicon semiconductors, but III-V compound semiconductors play a crucial role in optoelectronic and high-frequency devices, superconductors are used in highly sensitive magnetic field sensors, and metals, oxide layers, and even polymers are used in the passive parts of circuits (e.g., the wiring and the dielectric interlayer). In all these fields, and irrespective of the material class, nanostructures are becoming more and more important, which is expressed in the term "nanoelectronics."

Nanoelectronic devices will be strongly affected by defect structures, including the grain-boundary structures, in their semiconductor and insulator materials because a small number of point defects will affect the electrical properties of a nanosized crystal more than they will those of the microsized crystals used in conventional electronic devices. ${ }^{1)-3)}$

Nonvolatile memory (NVM) in the form of flash memory has already become an indispensable technology in portable and mobile electronic devices such as cellular phones, digital cameras, personal digital assistants, and notebook computers. These devices are going to handle huge amounts of digital data and therefore need high-speed, high-density NVMs that are inexpensive, reliable, and consume little power. One of the candidate next-generation nonvolatile memories that can replace flash memory is resistive random access memory (RRAM) ${ }^{4), 5)}$ Its simple structure consists of two terminal electrodes sandwiching a piece of material whose resistance changes when a current or voltage pulse is applied to the material. A small bias voltage senses the resistance of the memory, and, if the system is disconnected from the electric circuit by a transistor, the resistance remains stable without being refreshed. The memory cell is thus represented as a 1T1R (1 transistor and 1 resistor) combination. ${ }^{5)}$ The simple planar structure of this kind of memory cell reduces not only its process cost but also its cell size. The principle of a RRAM cell is similar to the reversible electrical switching in disordered structures that was described in $1968^{6}$ in that it is a resistance change triggered by an electric pulse, but the resistance change in a RRAM cell results from a phase change between the amorphous and crystalline states. This feature is mainly based on the carrier with the lower mobility and the carrier with the lower concentration in the amorphous phase of ternary alloy such as GeSbTe. The phase-change alloy can be heated by current flow, which makes it possible to write information into memory cell electronically. The same principle is used for optical data storage: a short strong pulse to amorphize the alloy, and then a longer less intense pulse to crystallize it. Materials that enable more rapid processing and have low writing powers and suitable resistances need to be developed. Although the crystal structure and stoichiometry of binary oxides that exhibit resistive change $\left(\mathrm{NiO}, \mathrm{TiO}_{2}\right.$, $\mathrm{ZnO}$, etc.) are more easily controlled than those of perovskite oxides, ${ }^{4), 5), 7)-11)}$ it has recently been reported that analogous heating of the bit material by a current flow similar to that in soft breakdown of insulating materials is the predominant step in forming a filament path before the switching action in binary-oxide-based RRAMs. ${ }^{12)-15)}$

The bit material of a RRAM cell, on the other hand, can be either an insulator or a semiconductor. Although the nature if the resistivity change produced by applying a unipolar or bipolar voltage pulse to an insulator has not yet been identified in detail, it has been proposed to be the formation of a filament path of nanodomain switches. ${ }^{16), 17)}$ The resistivity change produced by applying a bipolar voltage pulse to a semiconductor is thought to be due to a Schottky barrier formed at the metal-semiconductor interface with deep-level traps. ${ }^{9)}{ }^{10)}$ And the space-charge-limited current with deep-level traps depends on the semiconductor bit material. ${ }^{11)}$ Accordingly, the features of both mechanisms depend on the nature of deep-level traps at the interface and on the work function of the metal used as the electrode.

In this review, we survey resistivity-change phenomena in both of the two types of bit materials for RRAM devices (insulators and semiconductors) and also propose high-speed resistivity-switching mechanisms based on electron transport mechanisms originated from the specific nanostructure with defects formation. 


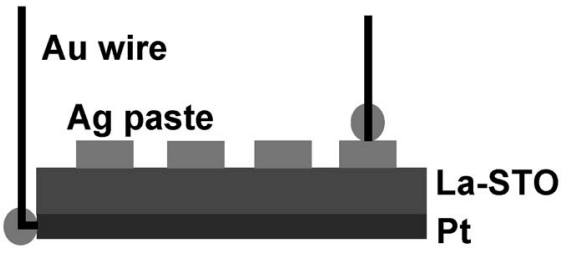

Fig. 1. Schematic structure of $\mathrm{Ag} / \mathrm{La}-\mathrm{STO} / \mathrm{Pt}$ specimen.

\section{Technical details}

We evaluated different types of resistivity-changing bit materials. The first was a $10 \times 10 \times 1.0-\mathrm{mm}$ piece of mirrorpolished (100) $0.75 \mathrm{wt} \%$-La-doped semiconductive $\mathrm{SrTiO}_{3}$ single-crystal (La-STO) (Furuuchi Chemical Co. Ltd.) exhibiting metallic semiconductivity $\left(N_{\mathrm{d}}=4 \times 10^{20} / \mathrm{cm}^{3}\right)$. A Pt bottom electrode was formed on the cleaned surface of the crystal by sputtering. Top electrodes $1.0 \mathrm{~mm}$ in diameter were formed by printing with epoxy resin Ag paste (typeDM6030Hk, Diemat Co., Ltd., Byfield, MA, U.S.A.) and drying at $200^{\circ} \mathrm{C}$ for $30 \mathrm{~min}$. The top and bottom electrode were then connected to the Au pad of an alumina substrate by gold wire and Ag paste as shown in Fig. 1.

The second resistivity-changing material we evaluated was a manganite perovskite $\left(\mathrm{Pr}_{0.7} \mathrm{Ca}_{0.3}\right) \mathrm{MnO}_{3}$ (PCMO). ${ }^{18)-20)} \mathrm{A} \mathrm{Si}$ substrate with an $\mathrm{SiO}_{2}$ layer and a $\mathrm{TiO}_{2}$ layer was heated to $400^{\circ} \mathrm{C}$ and 250 -nm-thick comb-shaped Pt bottom electrodes were formed by sputtering in a $100 \% \mathrm{Ar}$ atmosphere. Various sizes of Pt contact plugs $\left(1 \mu \mathrm{m}^{2}, 2 \mu \mathrm{m}^{2}, 5 \mu \mathrm{m}^{2}\right.$ and $\left.10 \mu \mathrm{m}^{2}\right)$ in the $\mathrm{SiO}_{2}$ insulating layer were formed on the bottom electrodes by using conventional CMOS (complementary metal oxide semiconductor) technology. The PCMO thin films (about 200-nm-thick) were fabricated on the Pt electrode by ablating various stoichiometric and nonstoichiometric $\left[\left(\operatorname{Pr}_{0.7} \mathrm{Ca}_{0.3}\right)_{1.5}\right.$ $\mathrm{MnO}_{3}$ and $\left.\left(\mathrm{Pr}_{0.7} \mathrm{Ca}_{0.3}\right)_{0.8} \mathrm{MnO}_{3}\right]$ ceramic targets with an ArF excimer laser on the substrates in a 20-mTorr oxygen atmosphere at various substrate temperatures between $300^{\circ} \mathrm{C}$ and $700^{\circ} \mathrm{C}$. A Pt top electrode on the PCMO film was then formed by sputtering (Fig. 2). The films deposited at temperatures other than $600^{\circ} \mathrm{C}$ were annealed at $600^{\circ} \mathrm{C}$ for 20 minutes in a 100-mTorr oxygen atmosphere in order to improve their crystallinity.

The third resistivity-changing material we evaluated was a surface-oxidized TiN diffusion-barrier metal. ${ }^{21,22)}$ A 40-nmthick TiN diffusion-barrier metal layer was formed on a $\mathrm{Pt} /$ $\mathrm{Ti} / \mathrm{SiO}_{2} / \mathrm{Si}$ substrate. After the top surface was oxidized at $400^{\circ} \mathrm{C}$ in a chamber with an oxygen atmosphere, the top $\mathrm{Pt}$ electrode was covered by a patterned mask of a resist film and both the electrode and the TiN layer were dry-etched (using a conventional photolithography technique) to form isolated memory cells less than $1 \mu \mathrm{m}$ in diameter. Then the $\mathrm{SiO}_{2}$ insulation layer was deposited by chemical vapor deposition, and the contact holes for the top Pt electrode and bottom Pt electrode of each memory cell filled with $\mathrm{TiN} / \mathrm{Al}-\mathrm{Si}-\mathrm{Cu} / \mathrm{TiN}$ were fabricated using CMOS technology (Fig. 3).

The resistive switching and measurement of the resistivity changes in the specimen were performed using a semiconductor parameter analyzer (Agilent 4156B or KEITHLEY 4200) and an oscilloscope connected to a high-speed pulse generator (Agilent 33250A). The resistivity change produced by applying a high-speed pulse with a potential less than $10 \mathrm{~V}$. was measured by applying $+0.7 \mathrm{~V}$, which was too low a voltage to induce resistance switching in the specimen. The capacitances of the high-resistivity and low-resistivity states of specimen

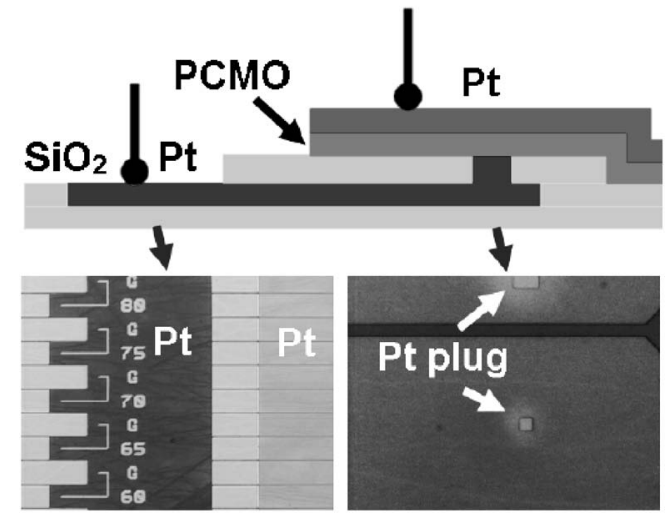

Fig. 2. Schematic structure of $\mathrm{Pt} / \mathrm{PCMO} / \mathrm{Pt}$ specimen.

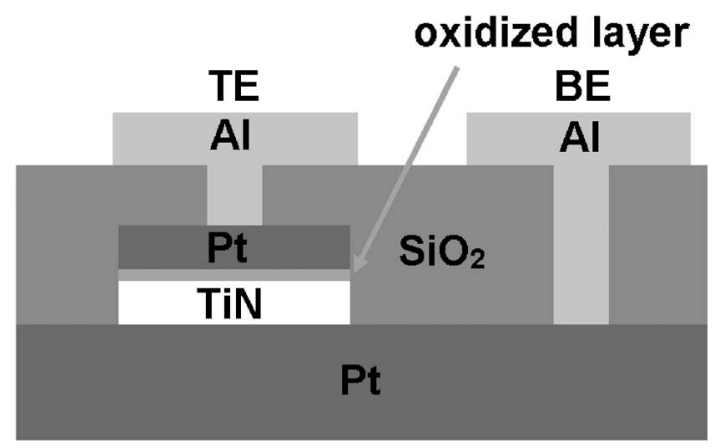

Fig. 3. Schematic structure of $\mathrm{Pt} / \mathrm{TiO}_{2} / \mathrm{TiN} / \mathrm{Pt}$ specimen.

were measured by an LCR meter (Agilent $4284 \mathrm{~A}$ ) at $100 \mathrm{kHz}$ with $V_{\mathrm{p}-\mathrm{p}}=0.45 \mathrm{~V}$. These electrical measurements were made at room temperature.

The crystallographic quality of the films was evaluated using an X-ray diffractometer (XRD) (MRD, Philips, Eindhoven, the Netherlands). Transmission electron microscopy (TEM) specimens were prepared using a focused ion beam. The cross-sectional nanostructure of the specimens was observed and their composition analyzed by using a scanning transmission electron microscope (STEM) (Topcon 002BF) with an energy dispersive spectroscopy (EDS) system (NORAN system six) and by electron energy loss spectroscopy (EELS) (GATAN GIF2000). The crystallographic features of the high-resolution TEM images obtained were identified by using selected area electron diffraction and fast Fourier transforms (FFT).

\section{Resistive switching of Ag/La-STO/Pt system}

The $I-V$ characteristic of $\mathrm{Ag} / \mathrm{La}-\mathrm{STO} / \mathrm{Pt}$ specimen was shown in Fig. 4. The sequence of increasing and decreasing the applied voltage is indicated by arrows. It can be seen that the $I-V$ characteristic shows hysteresis and rectification. It is suggested that the bottom Pt electrode does not have a rectifying property but the top Ag electrode does. Pt electrode has a larger work function $(5.4 \mathrm{eV})$ than $\mathrm{Ag}(4.3 \mathrm{eV}),{ }^{23)}$ but for contacts with highly doped STO the barrier width becomes very narrow, and tunneling current becomes dominant and shows ohmic contact properties. ${ }^{24)}$ The cross-sectional TEM image of $\mathrm{Ag}$ paste electrode is shown in Fig. 5. It can be deduced that the $\mathrm{Ag}$ particles surrounded by resin are in point-contact with the STO surface. Therefore, the rectifying 


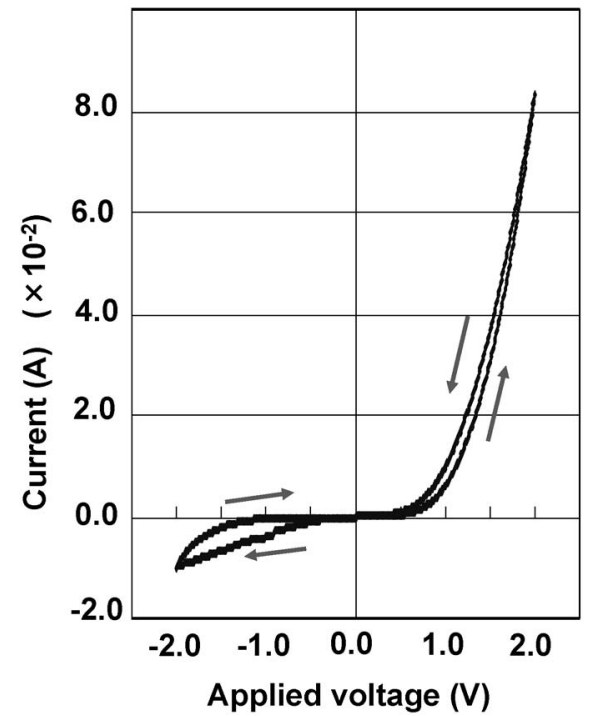

Fig. 4. $I-V$ characteristic of $\mathrm{Ag} / \mathrm{La}-\mathrm{STO} / \mathrm{Pt}$ specimen.

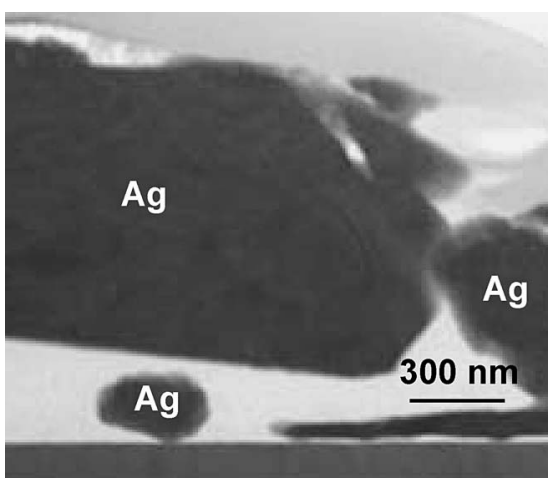

Fig. 5. Cross-sectional TEM image of the Ag paste electrode.

property of $\mathrm{Ag} / \mathrm{STO}$ interface is similar to that of a point-contact rectifier. The $I-V$ characteristics are actually those of a point-contact rectifier, whose forward and reverse $I-V$ characteristics are usually not as good as those of a planar Schottky diode. ${ }^{25)}$ The $I-V$ characteristics of this sample are also difficult to predict from theory, since its rectifying property is subject to wide variations due to variations in the $\mathrm{Ag}$ grain pressure, contact area, surface treatment and formation processes. Its large leakage current due mainly to the surface effect that reduces the rectification ratio, and its soft reverse breakdown characteristics are due to a large concentration of field beneath the metal point.

The pulse-induced resistance changes of the specimen were measured at several of the Ag top electrodes. They all exhibited bipolar resistive switching, though their initial resistance differed from electrode to electrode, perhaps because of the abovementioned wide variations of the point contacts. Bipolar resistive switching measured by applying single positive $7 \mathrm{~V}$ amplitude 500-ns-wide pulse and single negative $7 \mathrm{~V}$ amplitude 500-ns-wide pulse alternatively to one of the Ag top electrode is shown in Fig. 6(a). Applying the positive pulse decreased the resistance by about $1 \mathrm{M} \Omega$, and applying the negative pulse restored the initial high-resistance state. After several such small resistance changes, the resistance of high-resistance state decreased to about a tenth that of the low-resistance state

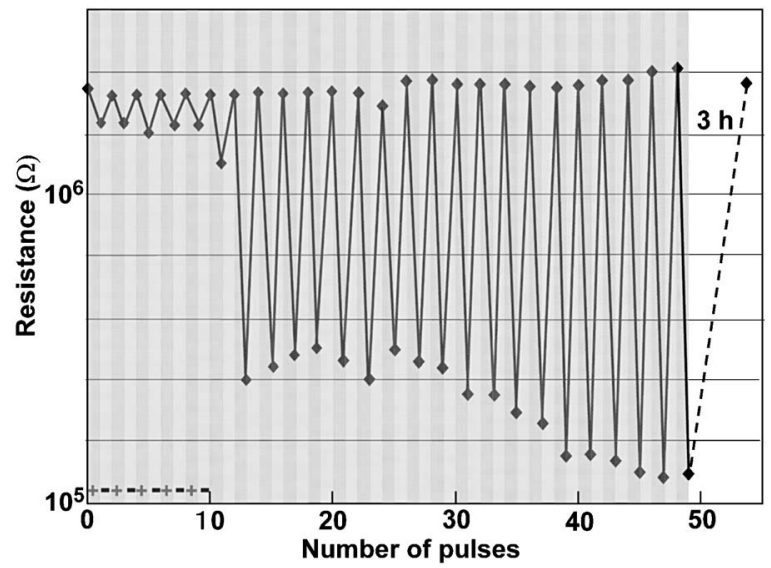

Fig. 6(a). Single-bipolar-pulse-induced resistive switching of a $\mathrm{Ag}$ / La-STO/Pt specimen and the retention of the resistivity if the switched state.

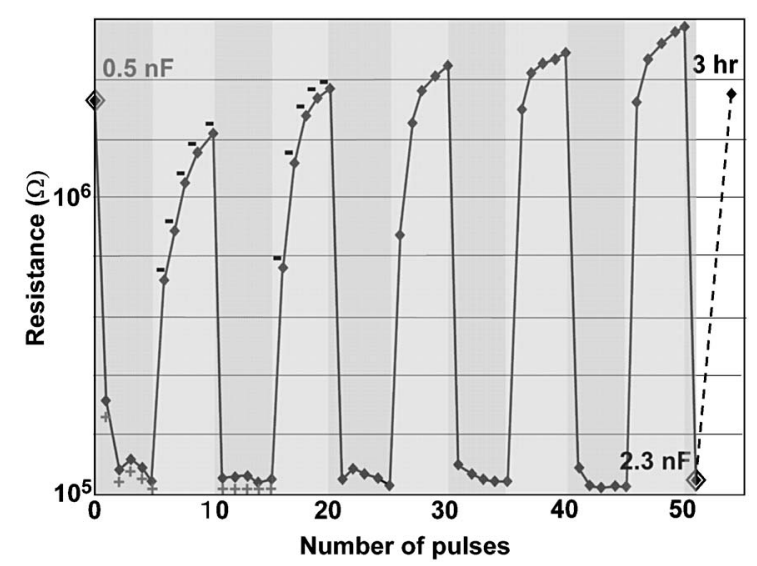

Fig. 6(b). Multiple-bipolar-pulse-induced resistive switching of a $\mathrm{Ag} / \mathrm{La}-\mathrm{STO} / \mathrm{Pt}$ specimen and the retention of the resistivity of the switched state.

before the negative pulse restored the high-resistance state. As this bipolar resistive switching was continued, however, the resistance of the high-resistance state gradually increased with of the number of pulses as that of the low-resistance state more markedly decreased. Finally, the bipolar resistive switching was stopped in the low-resistance state, and the resistance was measured again 3 hours later to see if it was still low. The resistance had, however, recovered its initial high value, suggesting that the resistive switching is transient phenomenon due to the Ag/ La-STO point-contact interface structure.

When five pairs of positive and negative pulses $( \pm 7 \mathrm{~V}$, each lasting $500 \mathrm{~ns}$ ) were alternately applied to the same Pt top electrode (Fig. 6(b)), the resistance of high-resistance state (reset) increased slightly with each positive pulse. The lowresistance state was measured again $3 \mathrm{~h}$ after the resistive switching measurement. Applying the multiple positive pulses, the resistance decreased from $3.3 \times 10^{6} \Omega$ to $1.3 \times 10^{5} \Omega$. And even a initial couple of pulses could not reach to the value. But during the resistive switching operations the last positive pulse of the multiple pulses always reached to the specific lowresistance value stably as if the value is a sort of saturated value. On the other hand, the resistance of high-resistance state increased gradually increased with the number of pulses. 

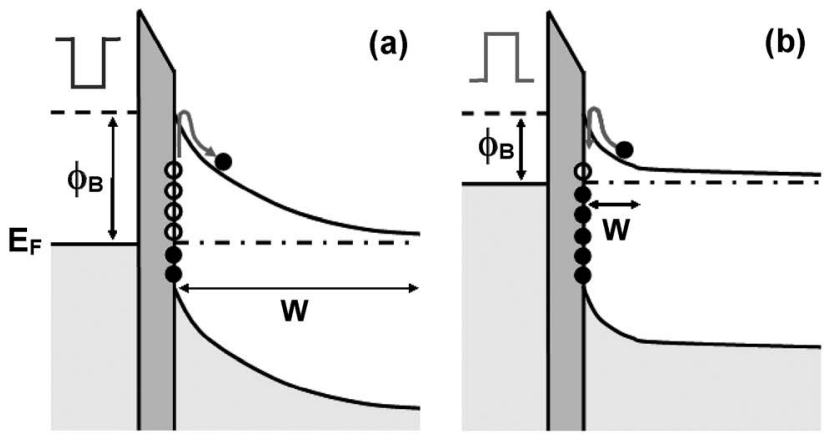

Fig. 7. Schematic illustration of the voltage-pulse-induced change in the barrier height of a specimen: (a) high-resistance state produced by applying a negative voltage pulse to the Ag electrode, and (b) lowresistance state produced by applying a positive voltage pulse to the Ag electrode.

The highest resistance value seemed to be saturated during applying multiple negative pulses, though, after switching by applying multiple positive pulses the resistance further increased with an increase of number of pulses over the initial high resistance value. After $3 \mathrm{~h}$ at room temperature, just as in the single bipolar pulse switching case the resistance of low-resistance state had become equal to that of the initial high-resistance state. The capacitance of high-resistance and low-resistance states of the specimen were measured during resistive switching measurement, and the capacitances of the initial high-resistance state and the final low-resistance state were respectively 0.5 and $2.25 \mathrm{nF}$. This capacitance difference between the high-resistance and low-resistance states indicates the changes of depletion layer thickness due to changes of the interface states of point contact interface of Ag to La-STO. Even though the interface is not like an ideal planar type of Schottky barrier, the possible schematic interfacial trap state change of specimen during the resistive change can be illustrated as shown in Fig. 7. The point contact is assumed not to be rigid contact but to be soft contact covered and pressured by insulative epoxy resin. An insulating layer at the interface can therefore be supposed. The potential barrier height $\phi_{\mathrm{b}}$ decreases with an increase in the number of electrons trapped at the interface states when they are injected by applying the positive pulse to the $\mathrm{Ag}$ electrode (Fig. 7(b)). This produces the low-resistance state. The recovery of the high-resistance state can be described as is follows. The potential barrier height $\phi_{\mathrm{b}}$ increases with a decrease of a number of trapped electrons at the interface states when those electrons are extracted by applying the negative pulse to the $\mathrm{Ag}$ electrode (Fig. 7(a)). The initial high-resistance state could represent a thermal equilibrium condition of the interface states. The reverting to the initial high-resistance state of the lowresistance state and the high-resistance state exhibiting higher resistance value compared with the initial high-resistance state can be understood as reverting to the thermally equilibrium condition of the interface states. It can be concluded that the bipolar resistive switching of Ag-paste/La-STO/Pt specimen is a result of transient interface state changes produced by applying voltage pulses; that is, a result of the injection and extraction of electrons at the interface state induced by applying pulses.

\section{Resistive switching of $\mathrm{Pt} / \mathrm{PCMO} / \mathrm{Pt}$ system}

Figure 8 shows the deposition-temperature dependence of the resistivity of PCMO thin films and also shows the resistivi-

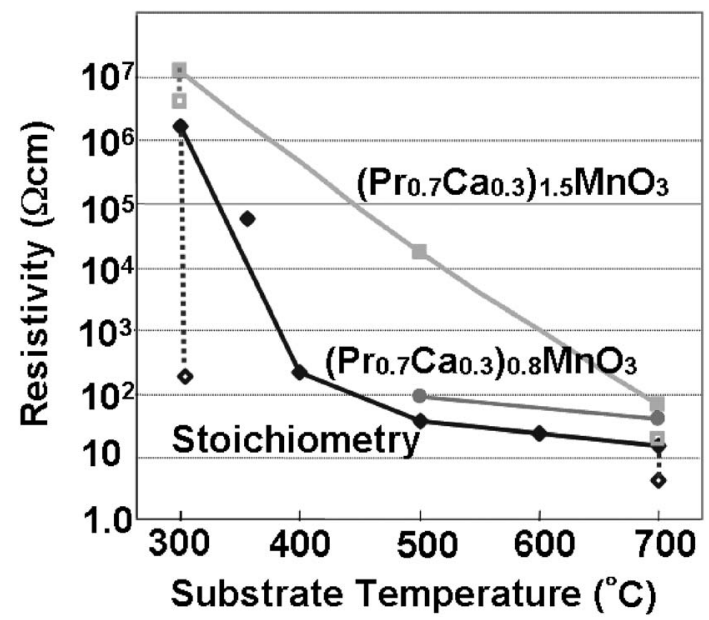

Fig. 8. Deposition-temperature-dependence of specimen resistance. The effect of oxygen annealing is also shown (open square represents annealed specimen).

ty changes after annealing. A $1-\mu \mathrm{m}^{2}$ Pt contact plug was used for the measurements in order to obtain higher resistivity. Specimens with stoichiometric composition showed an abrupt decrease in resistivity from $200 \mathrm{k} \Omega \mathrm{cm}$ at $300^{\circ} \mathrm{C}$ to $20 \Omega \mathrm{cm}$ at $400^{\circ} \mathrm{C}$. Furthermore, the resistivity gradually decreased with increasing deposition temperature. The resistivities of the nonstoichiometric specimens deposited at any temperature were higher than those of the stoichiometric specimens deposited at the same temperature, but the resistivity of both $\left(\operatorname{Pr}_{0.7} \mathrm{Ca}_{0.3}\right)_{1.5}$ $\mathrm{MnO}_{3}$ and $\left(\mathrm{Pr}_{0.7} \mathrm{Ca}_{0.3}\right)_{0.8} \mathrm{MnO}_{3}$ deposited at $700^{\circ} \mathrm{C}$ was less than $10 \Omega \mathrm{cm}$. As a result, we could not produce a specimen with high resistivity when using a high deposition temperature, which can produce high-crystallinity thin films. The low resistivity of bulk single-crystal PCMO specimens at room temperature $(\approx 0.5 \Omega \mathrm{cm})$ has already been reported. ${ }^{26), 27)}$ It has also been reported that the resistivity of epitaxially grown $\left(\operatorname{Pr}_{1-x} \mathrm{Ca}_{x}\right) \mathrm{MnO}_{3}$ thin films with $x \neq 0.3$, measured at room temperature using a four-probe method with $\mathrm{Ag}$ and $\mathrm{Pt}$ contact pads, was less than $0.01 \Omega \mathrm{cm} .^{28)-31)}$ Given the high crystallinity of epitaxially grown thin films and single crystals, our results are not inconsistent with the previously reported resistivities of PCMO specimens.

Using an XRD, we then investigated the relation between the deposition temperature and the crystallinity of specimens. Figure 9 shows the $\theta-2 \theta$ profiles of the stoichiometric thin films deposited at various temperatures. The films deposited at $600^{\circ} \mathrm{C}$ and $700^{\circ} \mathrm{C}$ exhibited PCMO (200)/(112) reflection peaks accompanied by a strong $\mathrm{Pt}$ (111) reflection peak. The full-width at half-maximum (FWHM) of the (200)/(112) peaks of the films deposited at $600^{\circ} \mathrm{C}$ and $700^{\circ} \mathrm{C}$ were both $0.38^{\circ}$, indicating that the films had good crystallinity even though no $(200) /(112)$ peaks were observed in the profiles of films deposited at temperatures below $500^{\circ} \mathrm{C}$. Scan profiles of $\psi$ from each specimen were obtained with $2 \theta$ fixed to $33^{\circ}$ in order to avoid strong reflections (Fig. 10). There were no peaks in the $\psi$ scan profiles of the specimens deposited at $300-400^{\circ} \mathrm{C}$ (the $\psi$ scan profiles of specimens deposited at $300^{\circ} \mathrm{C}$ and $350^{\circ} \mathrm{C}$ are not shown in Fig. 10). The thin films deposited at temperatures below $400^{\circ} \mathrm{C}$ were amorphous. In the $\psi$ scan profile of the specimen deposited at $500^{\circ} \mathrm{C}$ there was a (102) reflection peak but no (100) peak. A (100) peak and a (102) peak appeared in the $\psi$ scan profile of the specimen deposited at $600^{\circ} \mathrm{C}$, and there was a (100) peak in the $\psi$ 


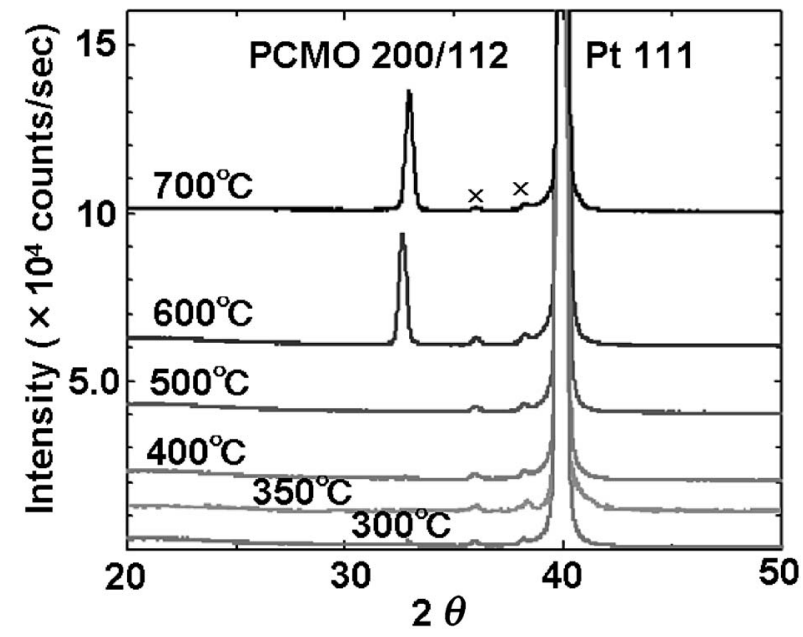

Fig. 9. $\theta-2 \theta$ profiles of thin films deposited at various temperatures. Small peaks indicated by " $x$ " are reflections from the $\mathrm{TiO}_{2}$ layer beneath the Pt bottom electrode.

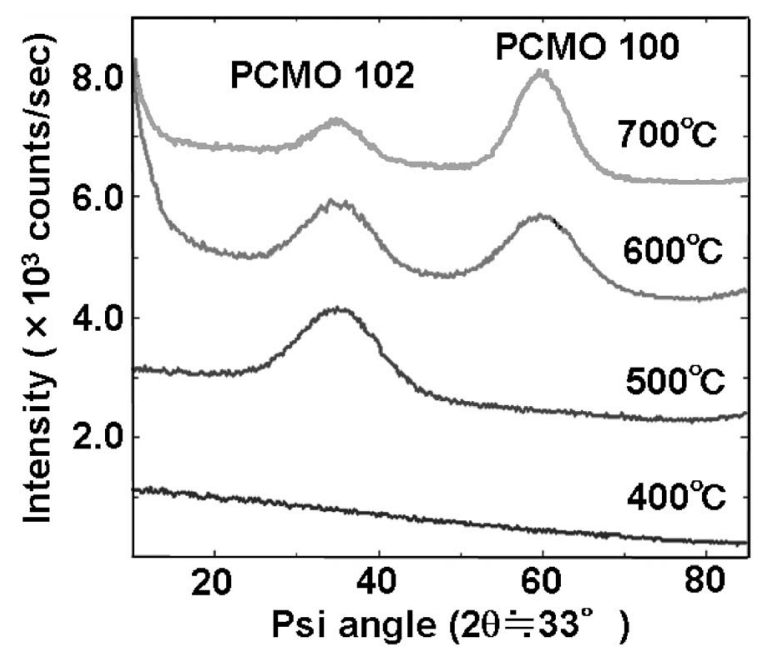

Fig. 10. $\psi$ scan profiles of the thin films. $2 \theta$ was fixed at $33^{\circ}$.

scan profile of the specimen deposited at $700^{\circ} \mathrm{C}$. These results show crystallization and crystal orientation of deposited films, that is, detectable crystallization of the film started at $500^{\circ} \mathrm{C}$ and (102) orientation occurred more often at the lower deposition temperature, while (100)/(112) orientations occurred more often at the higher deposition temperature.

Figure 11 shows a cross-sectional TEM image of the PCMO thin film deposited on a Pt electrode at $600^{\circ} \mathrm{C}$. It can be seen that columnar PCMO grains about $20 \mathrm{~nm}$ in diameter were grown on a flat surface of the polycrystalline Pt electrode. The selected area diffraction (SAD) patterns obtained from one grain of the Pt electrode and at least three different PCMO grains are shown in Fig. 12. Each of the diffraction spots was indexed by using the previously reported lattice parameter data for PCMO. ${ }^{32)}$ These SAD patterns show crystallographic orientations of $\mathrm{PCMO}(200)$ and $\mathrm{PCMO}(112)$ on a $\mathrm{Pt}(111)$ grain. It must be remarked that $\operatorname{PCMO}(200)$ and (020) diffraction spots cannot be distinguished by a conventional TEM. That is, the difference between the $a$-axis and $b$-axis cannot be distinguished by a TEM. In this paper therefore, PCMO (200) diffraction represents both $\operatorname{PCMO}(200)$ and

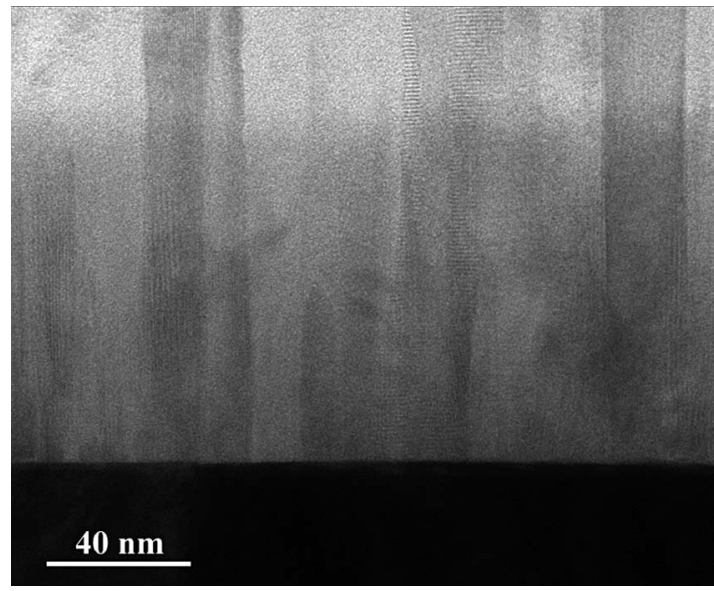

Fig. 11. Cross-sectional TEM image of a columnar-structured PCMO thin film deposited on a Pt electrode at $600^{\circ} \mathrm{C}$.

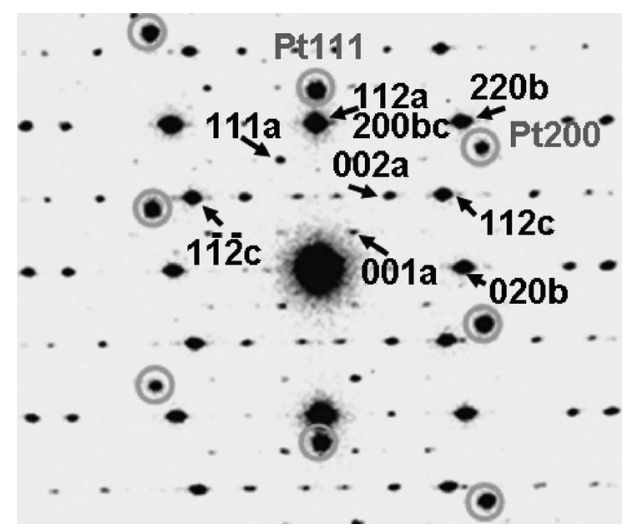

Fig. 12. Selected area electron diffraction pattern from the PCMO grains and a Pt grain in the film shown in Fig. 11. At least three different PCMO grains ( $a$ " and " $b$ " and " $c$ ") could be identified by indexing the diffraction spots, and three of them on the $\mathrm{Pt}(111)$ grain are oriented to (200) or (112).

(020), and $\mathrm{PCMO}[100]$ diffraction represents both PCMO $[100]$ and $[010]$. Because the only information about specific crystallographic orientations that was obtained from the cross-sectional TEM image (Fig. 11) was the surface normal and the restricted number of PCMO columns, we used planview TEM images to characterize the precise in-plane orientation relations between the (111) Pt grain and the (200), (112), and (102) PCMO grains. We can see in Figs. 13(a) and (b), which show plan-view images of the Pt electrode and the PCMO thin film, that the Pt grains are 100 to $300 \mathrm{~nm}$ in diameter and the PCMO grains are 20 to $40 \mathrm{~nm}$ in diameter. Thus it has been confirmed that the Pt electrode is mainly composed of columnar crystal grains averaging about $200 \mathrm{~nm}$ in diameter, each having the outermost surface corresponding to a self-orientation closest packed structure face (111), and that most of these columnar crystal grains are oriented to the (111) crystal plane. It can be deduced that the 10 -fold larger grain size of $\mathrm{Pt}$ enables the $\mathrm{Pt}$ to be a substrate for local epitaxial growth of PCMO, but these crystal grains have no in-plane crystallographic orientation relations with one another and the $\mathrm{Pt}$ electrode has a uniaxial-orientation polycrystalline structure. ${ }^{33)}$ A high-resolution TEM plan-view image of a thin film of PCMO grown on a (111)-oriented 


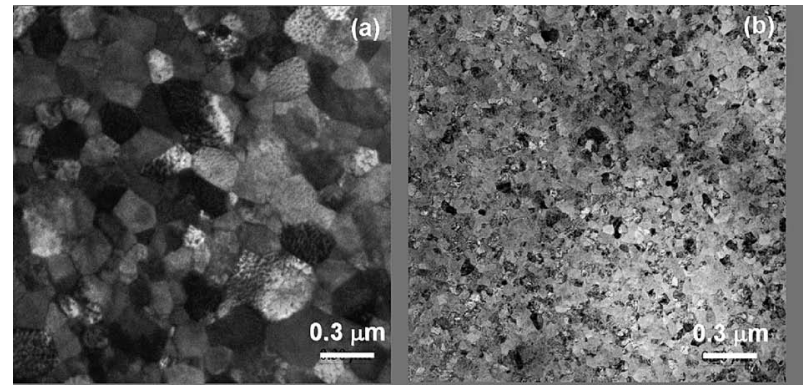

Fig. 13. Plan-view TEM images of (a) Pt electrode and (b) PCMO thin film.

grain of a Pt electrode is shown in Fig. 14(a), where the in-plane orientation of each numbered grain is indicated according to corresponding indexed FFT image shown Fig. 14 (b). The obtained projection plane of each grain can also indexed according to the pseudo-cubic form of PCMO: PCMO (112), PCMO (100) and PCMO (102) respectively correspond to $\mathrm{PCMO}(101) \mathrm{p}, \mathrm{PCMO}(110) \mathrm{p}$ and PCMO (111) $\mathrm{p}$ ( $\mathrm{p}$ denotes pseudo-cubic) ${ }^{19)}$ Of the 13 indexed PCMO grains, eight are (112) oriented, three are (100) oriented and two are (102) oriented. That the (102)-oriented grains were fewest is consistent with (102)-oriented grains not being observed in the cross-sectional TEM image and with the low intensity of PCMO (102) reflection in the $\psi$ scan profiles of the PCMO thin films deposited at $300^{\circ} \mathrm{C}$ and $600^{\circ} \mathrm{C}$. There are three different PCMO grains with specific projection planes and in-plane orientations: $\mathrm{PCMO}(112)$ with a $[-110]$ projection plane and $[11-1]$ in-plane orientation, $\operatorname{PCMO}(100)$ with a $[010]$ projection plane and $[001]$ in-plane orientation, and PCMO (102) with a [010] projection plane and [-110] in-plane orientation. The FFT image obtained from the whole image shown in Fig. 14(a) shows clear three-fold symmetry in-plane crystallographic relations between the PCMO grains, which indicates that semicoherent grain boundaries were formed in the columnar-structured thin film. It can also be seen from the FFT image obtained from the whole image in Fig. 14(a) that three of them could have the same type of three-fold symmetry corresponding to $\mathrm{Pt}(111)$ in-plane orientation. Even though most of the grains in the PCMO thin film exhibited PCMO (112) [-110][11-1] in-plane orientation, we can propose the following nine possible local epitaxial growth forms of PCMO (112), (100), and (102) on Pt(111): PCMO (112) $[11-1][-110] / / \operatorname{Pt}(111)[1-10][11-2], \quad \operatorname{PCMO}(112)$ $[11-1][-110] / / \operatorname{Pt}(111)[01-1][-211], \quad \operatorname{PCMO}(112)[11-1]$ $[-110] / / \mathrm{Pt}(111)[-101][1-21], \operatorname{PCMO}(100)[010][001] / / \mathrm{Pt}$ (111) $[-110][-1-12], \quad \operatorname{PCMO}(100)[010][001] / / \mathrm{Pt}(111)$ $[10-1][2-1-1], \quad \operatorname{PCMO}(100)[010][001] / / \mathrm{Pt}(111)[0-11]$ $[-211], \quad \operatorname{PCMO}(102)[010][-110] / / \mathrm{Pt}(111)[-110][11-2]$, $\mathrm{PCMO}(102)[010][-110] / / \mathrm{Pt}(111)[10-1][1-21]$ and PCMO (102) $[010][-110] / / \operatorname{Pt}(111)[0-11][-211]$.

Thus, local epitaxial growth with high-crystallinity threefold symmetry was confirmed in the PCMO deposited at a high temperature.

$\theta-2 \theta$ profiles of the specimens deposited at $300^{\circ} \mathrm{C}$ or $700^{\circ} \mathrm{C}$ and then annealed at $600^{\circ} \mathrm{C}$ in an oxygen atmosphere are shown in Fig. 15. The (200)/(112) reflection peak appeared in the profile of the specimen deposited at $300^{\circ} \mathrm{C}$. The annealing of the deposited films improved the crystallinity of the films and corresponds to the resistivity decrease shown in Fig. 8.

Reisistive switching measurements were carried out on all

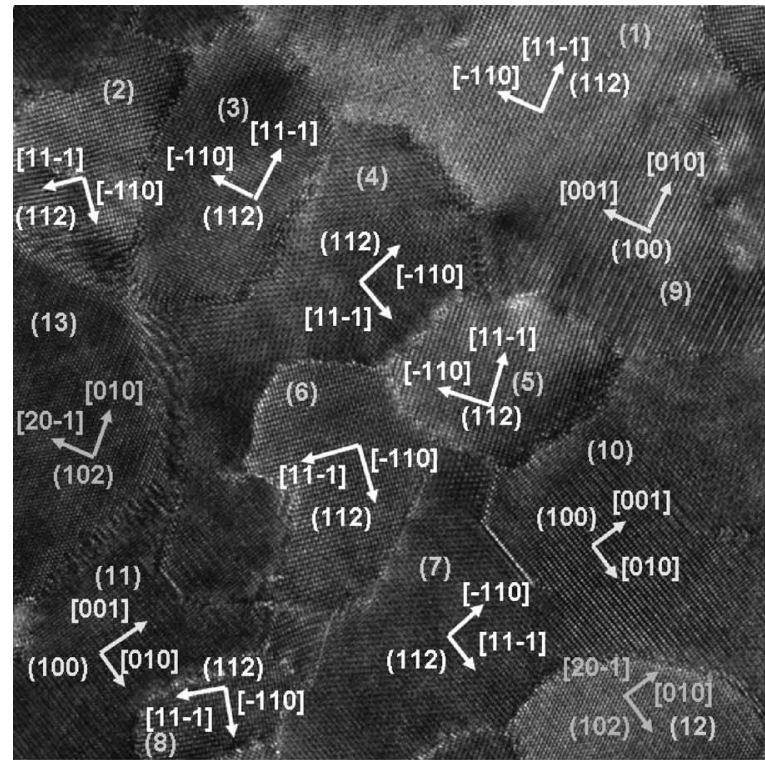

(a)

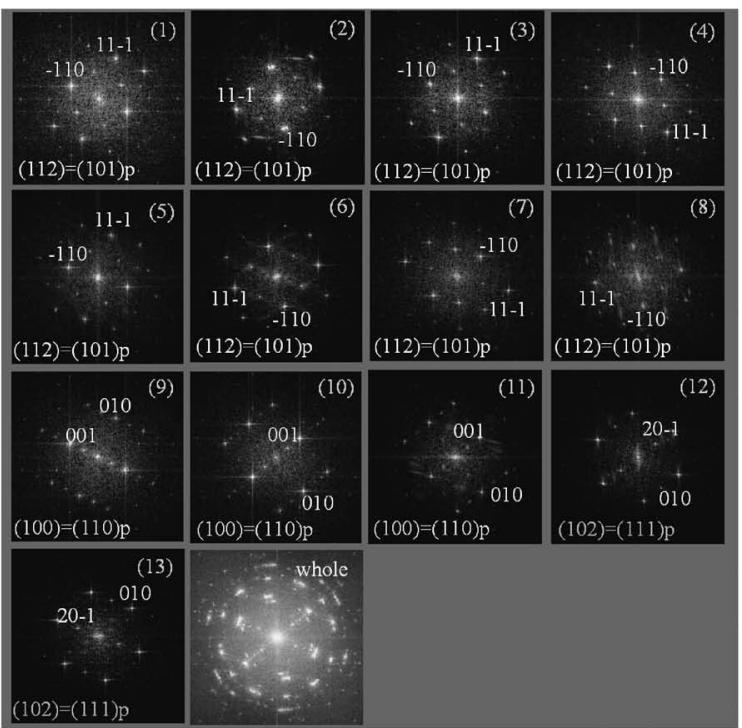

(b)

Fig. 14. (a) HR-TEM plan-view image of PCMO thin film grown on (111)-oriented grains of a Pt electrode. In-plane orientation indicated in each numbered grain corresponds to the indexed FFT images shown in part (b). (b) FFT images obtained from each of the numbered grains shown in part (a). The projection plane and corresponding pseudo-cubic perovskite projection plane are indicated. The last FFT image was obtained from the whole image shown in part (a).

the specimens. As can be expected, the low-resistivity specimens did not show any resistive switching when various differently shaped pulses were applied. And the high-resistivity specimens, the stoichiometric and $\left(\mathrm{Pr}_{0.7} \mathrm{Ca}_{0.3}\right)_{1.5} \mathrm{MnO}_{3}$ thin films deposited at $300^{\circ} \mathrm{C}$ and the $\left(\operatorname{Pr}_{0.7} \mathrm{Ca}_{0.3}\right)_{0.8} \mathrm{MnO}_{3}$ thin films deposited at $300^{\circ} \mathrm{C}$ and annealed at $300^{\circ} \mathrm{C}$, did not show resistive switching. Only the film deposited at $350^{\circ} \mathrm{C}$ exhibited monopolar resistive switching (Fig. 16). The initial resistance of $80 \mathrm{k} \Omega$ decreased to $8 \mathrm{k} \Omega$ when a single positive $3-\mathrm{V} 10-\mu \mathrm{s}$ pulse was applied. When a positive $5-\mathrm{V} 100-\mu$ s pulse was applied the thin film reverted to a low-resistance state. The change in the polarity of monopolar switching was produced by applying a negative $5-\mathrm{V} 100-\mu$ s pulse when the film was in a 


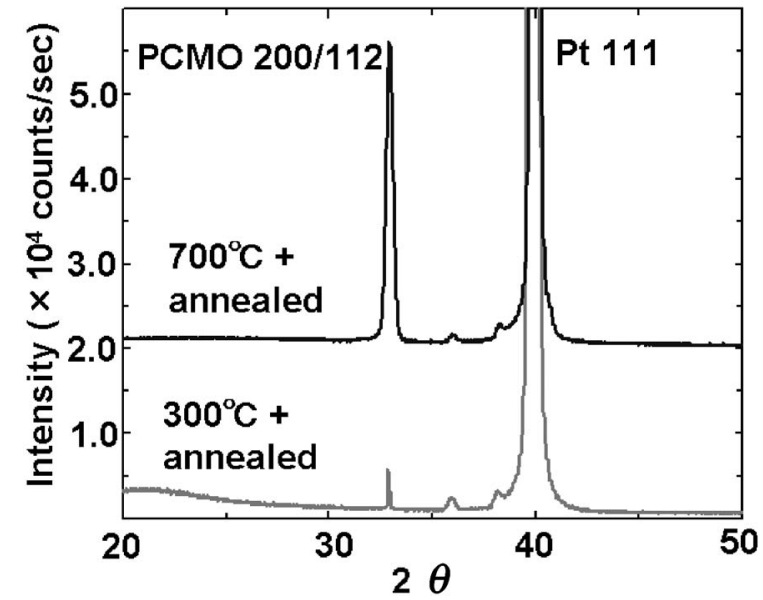

Fig. 15. $\theta-2 \theta$ profiles of the thin films deposited at $300^{\circ} \mathrm{C}$ and $700^{\circ} \mathrm{C}$ and then annealed.

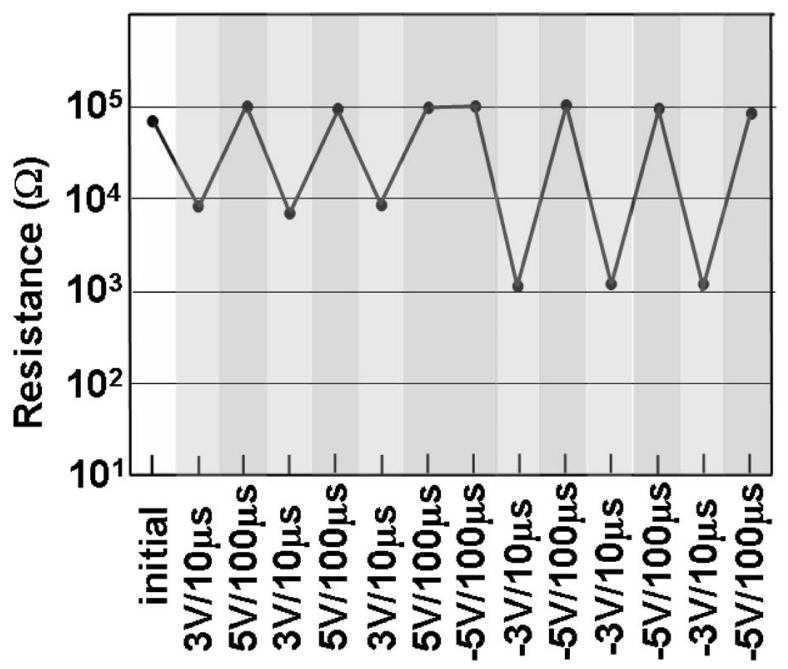

Fig. 16. Monopolar resistivity switching of the thin film deposited at $350^{\circ} \mathrm{C}$. Polarity of the monopolar switching can be changed by applying a reversed pulse.

high-resistance state. The following negative $3-\mathrm{V} 10-\mu$ s pulse switched the high-resistance state to a low-resistance state. The resistance of the film in the low-resistance state was one tenth that of the state produced by positive monopolar switching, and this low-resistance state was restored by applying a negative $5-\mathrm{V} 100-\mu$ s pulse. The active area of the PCMO thin film is determined by the size of the Pt contact plug, but the fabricated device structure (Fig. 2) tends to have stray capacitance between the top Pt electrode and the bottom Pt electrode. We deduced that this stray capacitance was the reason we had to apply pulses with widths of microsecond order in order to obtain resistive switching instead of the nanosecond-order pulses reported to elicit resistive switching. ${ }^{5), 34)}$ There is a possibility of resistive switching in other high-resistivity thin films if the stray capacitance does not influence the applying pulse. The polarity-switchable monopolar resistive switching might also be due to the stray capacitance, and bipolar switching might be possible if there were no influential stray capacitance.

We could not obtain high resistivity in all the high-crystallinity PCMO thin films fabricated in this study, or even in all those with nonstoichiometric composition. This indicates the absence of a Schottky barrier between the Pt electrode and the PCMO thin films deposited by pulsed laser deposition in an oxygen atmosphere. Amorphous thin films deposited at a low temperature $\left(350^{\circ} \mathrm{C}\right)$ showed monopolar resistive switching. Therefore, the previously proposed resistive switching model based on the Schottky barrier with a charge-trapping interface state formed when a p-type semiconductor PCMO comes into contact with a metal electrode is not applicable in this case. Phenomenologically, the model of filament paths formation with nanodomain switches is applicable to the observed resistive switching in the amorphous thin film in this study. There is, however, no clear evidence for the formation of filament paths and the presence of nanodomain switches. Many questions remain regarding previously reported PCMO thin films that exhibited crystallographic resistive switching related to the interfacial nanostructures adjacent to the electrode, including oxidation of the electrode itself, especially in cases where the electrodes were $\mathrm{Ag}$ and $\mathrm{Ti}$ adopted as the electrodes. Much research is required into nanostructural analysis of crystallographic features and the appropriate resistive switching mechanism, including the nanoscale of the Mott transition and the related strong correlation effects. ${ }^{35)}$

\section{Resistive switching of $\mathrm{Pt} / \mathrm{TiO}_{2} / \mathrm{TiN} / \mathrm{Pt}$ system}

After measurement and confirmation of the initial highresistance state $\left(8 \times 10^{4} \mathrm{ohm}\right)$, a single negative 2.0 -V 20 -ns pulse was applied to the device. The resistance of the lowresistance state was about $2 \times 10^{2} \Omega \mathrm{ohm}$, and the resistance change was almost $40000 \%$. The low-resistance state was restored to a high-resistance state by applying a positive $2.2-\mathrm{V}$ 30-ns pulse (Fig. 17). The measured resistance of the highresistance state was found to be almost equal to that of the initial high-resistance state. Thus, if an appropriate switching condition is chosen, it is not necessary for the specimen to have previously undergone filament path processing before the stable switching operation. ${ }^{12)-14), 16)}$

Figure 18 shows a cross-sectional STEM image of the specimen shown in Fig. 3 (the image was rotated $90^{\circ}$ ) and the EDS chemical composition line profile of the $\mathrm{Pt} / \mathrm{TiO}_{2} / \mathrm{TiN} / \mathrm{Pt}$ memory cell in the low-resistance state. The line profile was obtained by scanning with an electron beam about $0.5 \mathrm{~nm}$ in diameter and almost coincides with the fabricated memory cell structure. The region of titanium and oxygen adjacent to the top $\mathrm{Pt}$ electrode suggests the formation of $\mathrm{a} \mathrm{TiO}_{2}$ nanolayer at the surface of the TiN thin film. Some of the diffusive distributions of oxygen and titanium seemed to be affected by spreading the electron beam in the thin film specimen by use of nanometer electron probe. Therefore it can be deduced that the effective oxide layer thickness is less than $5 \mathrm{~nm}$. Thus, the formation of the oxidized layer on the TiN thin film was clearly confirmed.

Furthermore, nanoprobe EELS measurements were carried out at this region and their ELNES features were characterized. An electron beam about $0.5 \mathrm{~nm}$ diameter was used for this measurement and characterization. Figure 19 (a) shows a HR-TEM image indicating EELS analysis points from which were obtained the EELS profiles shown in Fig. 3(b). Arrows indicate ELNES of splitting into $2 p_{3 / 2}$ and $2 p_{1 / 2}$ levels (position "1" and "2") and further splitting into two maxima attributed to the crystal-field interaction (position " 3 "). In Fig. 19(b) the EELS profile obtained from point " 3 " is enlarged and compared with the previously reported EELS profiles of $\mathrm{TiO}_{2}$ rutile and anatase. ${ }^{36)}$ It is well known that the near-edge features of EELS reflect the symmetry-projected 


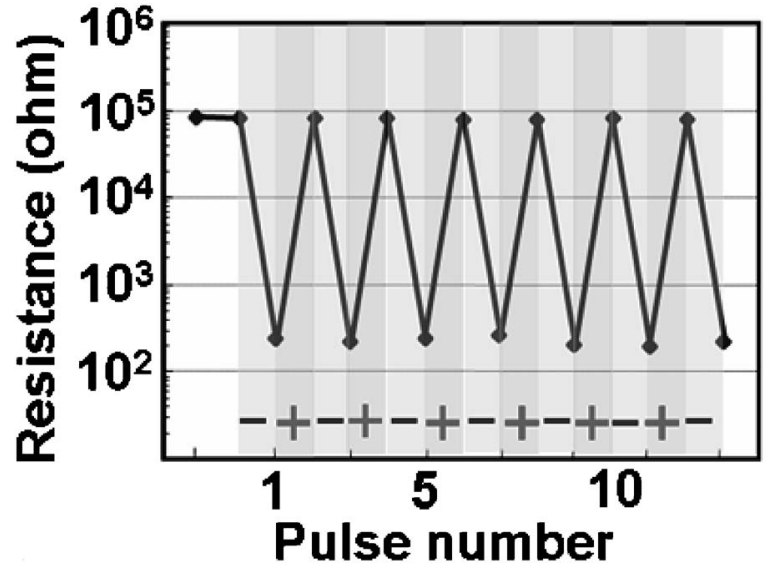

Fig. 17. Measured bipolar resistive switching of the fabricated device.

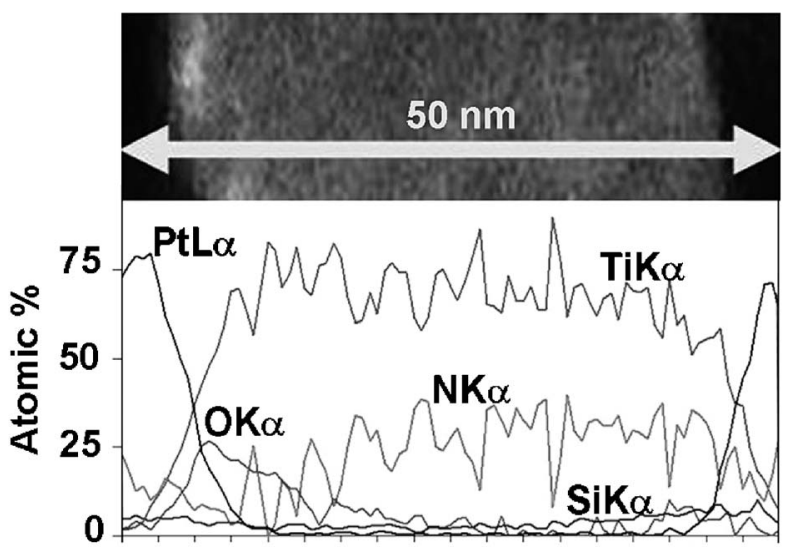

Fig. 18. Cross-sectional STEM image and EDS chemical composition profile of a $\mathrm{Pt} / \mathrm{TiO}_{2} / \mathrm{TiN} / \mathrm{Pt}$ memory cell. The scanning line is indicated by an arrow.

density of the unoccupied state in the simple single-electron picture. The conductive barrier metal TiN is a cubic structure and belongs to space group $F m 3 m$ (no. 225), whereas rutile and anatase both have tetragonal structures and respectively belong to space groups $P 4_{2} / \mathrm{mnm}$ (no. 136) and $I 4_{1} /$ amd (no. 141). The octahedron of $\mathrm{TiN}_{6}$ in the cubic TiN shows high symmetry without distortion, described as $O_{\mathrm{h}}$. On the other hand, the octahedron of $\mathrm{TiO}_{6}$ in the rutile shows a slight orthorhombic distortion, while that in the anatase is so significantly distorted that its symmetry is lower than orthorhombic. Specifically, the local point-group symmetry around the Ti atom is lowered from $O_{\mathrm{h}}$ to $D_{2 \mathrm{~h}}$ in rutile and from $O_{\mathrm{h}}$ to $D_{2 \mathrm{~d}}$ in anatase. The two lowest unoccupied orbitals, containing mostly Ti $3 d$ characteristics, separate into two groups, the threefold $t_{2 \mathrm{~g}}$ and the twofold $e_{\mathrm{g}}$ in the local symmetry of TiN, so the $2 p$ spin-orbit interaction splits into the $2 p_{3 / 2}$ and $2 p_{1 / 2}$ levels. In the same manner, the $2 p$ spinorbit interaction splits into the $2 p_{3 / 2}\left(L_{3}\right)$ and $2 p_{1 / 2}\left(L_{2}\right)$ levels with a separation of $5.4 \mathrm{eV}$ in both rutile and anatase, but these levels are further split into two maxima attributable to the crystal-field interaction, where the octahedral coordination of Ti atoms with oxygen splits the degenerate $3 d$ states into two states: $t_{2 \mathrm{~g}}\left(3 d_{\pi}\right)$ and $e_{\mathrm{g}}\left(3 d_{\sigma}\right)$. The shapes of the resultant spectra for rutile and anatase are generally similar,
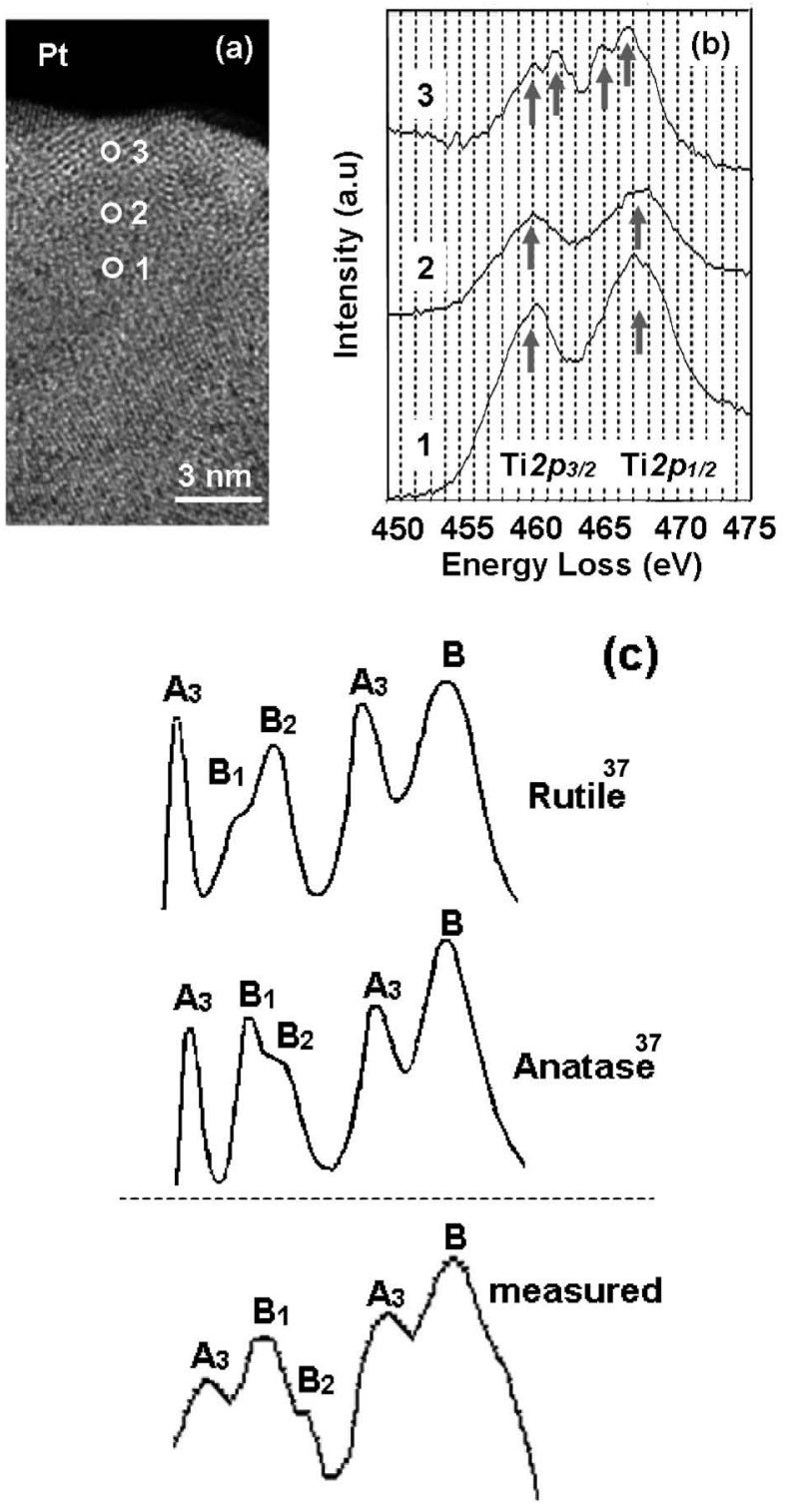

Fig. 19. (a) Cross-sectional HR-TEM image. EELS analysis positions are indicated by open circles. (b) EELS spectra obtained from the positions indicated by open circles in (a). Arrows indicate ELNES of splitting into the $2 p_{3 / 2}$ and $2 p_{1 / 2}$ levels in TiN and further splitting into two maxima attributed to the crystal-field interaction of $\mathrm{TiO}_{2}$. (c) Enlarged ELNES obtained from the position closest to the top $\mathrm{Pt}$ electrode (indicated as open circle number 3 in Fig. 19(a)). ELNES fingerprints of $\mathrm{TiO}_{2}$ anatase and rutile are also shown. ${ }^{36)}$

but a noticeable difference between these two crystals is observed on the second peak of the $L_{3}$ edge, $2 p_{3 / 2} 3 d_{\sigma}\left(\mathrm{B}_{1}\right.$ and $\mathrm{B}_{2}$ in the spectra), which for rutile appears as a low-energy shoulder and for anatase appears as a high-energy shoulder. ${ }^{36)}$ This shape difference of the $2 p_{3 / 2} 3 d_{\sigma}$ peak is thought to be due to the distortion of the Ti sited from octahedral to $D_{2 \mathrm{~h}}$ symmetry in rutile and to $D_{2 \mathrm{~d}}$ symmetry in anatase. ${ }^{36), 37)}$ Figures 19(a) and (b) clearly indicate changes in the nanostructure from TiN to $\mathrm{TiO}_{2}$ by the splitting of the $2 p_{3 / 2}$ and $2 p_{1 / 2}$ peaks, reflecting lower symmetry of $\mathrm{TiO}_{2}$ rutile and anatase. Less than $3 \mathrm{~nm}$ of the $\mathrm{TiO}_{2}$ oxidized layer adjacent to the top Pt electrode was revealed. Furthermore, the detail of ELNES obtained from point " 3 " shows that the oxidized layer is not rutile but anatase. Given the lower critical donor density 


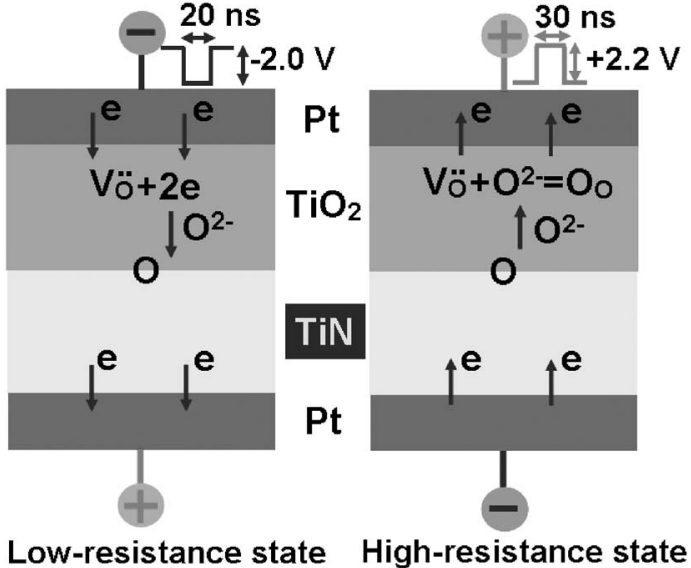

Fig. 20. Schematic picture of high- and low- resistivity states of a specimen.

of anatase for Mott transition, ${ }^{38)}$ the obtained results indicate that the high-speed resistive switching is a result of the Mott transition. The increase in donor concentration triggering the Mott transition could primarily be a result of the negative nanosecond pulse injecting electrons from the top electrode into the anatase monolayer, causing oxygen vacancies to form to form there as $\mathrm{O}^{2-}$ migrates to and adsorbs on the bottom electrode. A positive nanosecond pulse, on the other hand, could extract electrons from the top electrode and be accompanied by $\mathrm{O}^{2-}$ migration and oxygen vacancy annihilation in the film and by absorption of $\mathrm{O}^{2-}$ on the top electrode (Fig. 20). It can be easily deduced that a nanosecond pulse is quite sufficient to abruptly increase or decrease the donor concentration in the nanolayer. The nano-active layer adjacent to the top Pt electrode properly explains the switching polarity of the memory cell. TiN can contain significant amounts of oxygen as commented that the diffusion barrier mechanism was that of the "stuffed barrier" with grain boundaries stuffed with oxygen. ${ }^{39), 40)}$ The TiN thin film could work as an oxygen reservoir, and the asymmetrical bipolar pulse required for stable resistive switching could be derived from the small resistance of the TiN thin film (small resistance deforms nanosecond pulse). It seems that the TiN barrier metal film and the anatase nanolayer can respectively be analogous to previously proposed "filament paths" and "nanosized domains (switch)."17) Reported various different type of pulse form (amplitude and width) for resistive change in the oxide thin films could be mainly defined as the resistance and dielectric constant of "filament paths" and the volume of "nanosized domains."

\section{Summary}

We studied different types of pulse-induced-resistivitychange materials from nanostructural and nanoelectronics viewpoints.

(1) We revealed that an interfacial structure and resultant bipolar resistive switching properties of $\mathrm{Ag}$ paste electrode and $\mathrm{La}-\mathrm{STO}$ semiconductor in Ag paste/La-STO/Pt specimen. An $\mathrm{Ag}$ paste electrode has often been used as electrode on resistivity-changing materials, ${ }^{41)-44)}$ the structure of its interface with semiconductive materials had not been studied before. The interfacial structure formed a Schottky barrier was interpreted as point contact of Ag grains to semiconductor, also the existence of insulative epoxy resin at the interface was strongly suggested.

(2) We first observed monopolar resistive switching in a PLD-deposited high-resistivity amorphous PCMO thin film sandwiched by Pt electrodes. There is no evidence of a Schottky barrier contribution to the switching, but there is strong evidence for the formation of filament paths and the existence of reversible nanodomain switches.

(3) We used STEM/EDS and EELS analyses to characterize a $\mathrm{TiO}_{2}$ anatase oxidized layer less than $3 \mathrm{~nm}$ thick on the TiN barrier metal thin film of a RRAM memory cell. The results suggest that the nanosecond-order bipolar resistive switching of the fabricated memory cell stems from a Mott transition of the anatase nanolayer and imply that the filament path formation previously observed in binary transition metal oxides might be a result of nanometer-order crystallographic structural change or deformation.

Acknowledgements We thank Mr. Shinji Kobayashi, Mr. Yasunari Hosoi, Mr. Kazuya Ishihara, Mr. Shigeo Ohnishi, Dr. Nobuyoshi Awaya (Sharp Corporation), Mr. Yuji Nishi and Dr. Toshimasa Suzuki (Taiyo Yuden Company) for their research collaboration. We also thank Dr. Hiroshi Koyama, Mr. Masashi Konagai and Ms. Ayami Matsui for technical assistance. This research was partly supported by a Grant-in Aid for Science Research (B) (No. 17360324) from the Japan Society for the Promotion of Science.

\section{References}

1) G. Schmid, Nanostructured Materials, 6, 15-24 (1995)

2) H. Gleiter, Progress in Materials Science, 33, 223-315 (1989).

3) N. Ichinose, Y. Ozaki and S. Kashu, "Superfine Particle Technology," Springer-Verlag, New York (1992).

4) S. Q. Liu, N. J. Wu and A. Ignatiev, Appl. Phys. Lett., 76, 2749-2751 (2000).

5) W. W. Zhuang, W. Pan, B. D. Ulrich, J. J. Lee, L. Stecker, A. Burnaster, D. R. Evans, S. T. Hsu, M. Tajiri, A. Shimaoka, K. Inoue, T. Naka, N. Awaya, K. Sakiyama, Y. Wang, S. Liu, N. J. Wu and A. Ignatiev, International Electron Devices Meeting, Technical Digest, 193-196 (2002).

6) S. R. Ovshinsky, Phys. Rev. Lett., 21, 1450-1453 (1968).

7) A. Beck, J. G. Bednorz, Ch. Gerber, C. Rossel and D. Widmer, Appl. Phys. Lett., 77, 139-141 (2000).

8) Y. J. Watanabe, G. Bednorz, A. Bietsch, Ch. Gerber, D. Widmer and A. Beck, Appl. Phys. Lett., 78, 3738-3740 (2001).

9) A. Sawa, T. Fujii, M. Kawasaki and Y. Tokura, Appl. Phys. Lett., 85, 4073-4075 (2004).

10) T. Fujii, M. Kawasaki, A. Sawa, H. Akoh, Y. Kawazoe and Y. Tokura, Appl. Phys. Lett., 86, 012107 (2005).

11) A. Odogawa, H. Sato, I. H. Inoue, H. Akoh, M. Kawasaki and Y. Tokura, Phys. Rev. B, 70, 224403-224407 (2004).

12) I. Baek, G. M. Lee, S. S. Seo, M. J. Lee, D. H. Seo, D. Suh, S. J. Park, C. S. O. Park, H. S. Kim, I. K. Yoo, U-In Chung and J. T. Moon, International Electron Devices Meeting, Technical Digest, 587-590 (2004).

13) S. Seo, M. J. Lee, D. H. Seo, E. J. Jeoung, D. S. Suh, Y. S. Young, I. K. Yoo, I. S. Byun, I. R. Hwang, S. H. Kim, I. S. Byun, J. S. Kim, J. S. Choi and B. H. Park, Appl. Phys. Lett., 85, 5655-5357 (2004).

14) S. Seo, M. J. Lee, D. H. Seo, S. K. Choi, D. S. Joung, I. K. Yoo, I. S. Byun, I. R. Hwang, S. H. Kim and B. H. Park, Appl. Phys. Lett., 86, 093509 (2005).

15) B. J. Choi, D. S. Jeong, S. K. Kim, C. Rohde, S. Choi, J. H. Oh, H. J. Kim, C. S. Hwang, S. Z. Szot, R. Waser, B. Reichenberg and S. Tiedke, J. Appl. Phys., 98, 033715 (2005).

16) G. Dearnaley, A. M. Stoneham and D. V. Morgan, Rep. Prog. Phys., 33, 1129-1191 (1970).

17) M. J. Rosenberg, I. H. Inoue and M. J. Sanchez, Phys. Rev. Lett., 92, 178302 (2004).

18) M. Fujimoto, H. Koyama, S. Kobayashi, Y. Tamai, N. Awaya, Y. Nishi and T. Suzuki, Appl. Phys. Lett., 89, 243504 (2006). 
19) Y. Nishi, T. Suzuki, H. Koyama and M. Fujimoto, J. Cryst. Growth, 299, 63-69 (2007).

20) M. Fujimoto, H. Koyama, Y. Nishi, T. Suzuki, S. Kobayashi, Y. Tamai and N. Awaya, J. Am. Ceram. Soc., 90, 2205-2209 (2007).

21) M. Fujimoto, H. Koyama, Y. Hosoi, K. Ishihara and S. Kobayashi, Japan. J. Appl. Phys., 45, L310-312 (2006).

22) M. Fujimoto, H. Koyama, M. Konagai, Y. Hosoi, K. Ishihara, S. Ohnishi and N. Awaya, Appl. Phys. Lett., 89, 223509 (2006).

23) S. M. Sze, "Physics of Semiconductor Devices," WileyInterscience, New York (1969) p. 366.

24) S. M. Sze, "Semiconductor Devices: Physics and Technology," Wiley, New York (1989) p. 169.

25) S. M. Sze, "Physics of Semiconductor Devices," WileyInterscience, New York (1969) p. 415.

26) Y. Tomioka, A. Asamitsu, Y. Moritomo and Y. Tokura, J. Phys. Soc. Japan, 64, 3626-3630 (1995).

27) Y. Tomioka, A. Asamitsu, H. Kuwahara, Y. Morimoto and Y. Tokura, Phys. Rev. B, 53, R1689 (1996).

28) W. Preller, A. M. Haghiri-Gosnet, B. Mercey, Ph. Lecoeur, Ch. Simon and B. Raveau, Appl. Phys. Lett., 77, 1023-1025 (2000).

29) A. M. Haghiri-Gosnet, M. Hervieu, Ch. Simon and B. Raveau, J. Appl. Phys., 88, 3545-3551 (2000).

30) W. Prellier, Ch. Simon, M. Herview, B. Mercey, A. M. Haghiri-Gosnet and B. Raveau, Phys. Rev. B, 62, R16337 (2000).

31) L. V. Saraf, S. B. Ogale, Z. Chen, R. P. Godfrey, T.
Venkatesan and R. Ramesh, Phys. Rev. B, 62, R11961 (2000).

32) Z. Jirak, S. Krupicka, Z. Simsa, M. Dolouha and S. Vratislav, J. Magn. Magn. Mater., 53, 153-166 (1985).

33) K. Morito, T. Suzuki and M. Fujimoto, Japan. J. Appl. Phys., 40, 1310-1314 (2001).

34) A. Y. Baikalov, Q. Wang, B. Shen, B. Lorenz, S. Tsui, Y. Y. Sun, Y. Y. Xue and C. W. Chu, Appl. Phys. Lett., 83, 957-959 (2004).

35) M. Imada, A. Fujimori and Y. Tokura, Rev. Mod. Phys., 70, 1039-1263 (1998).

36) R. Brydson, H. Sauer, W. Engel, J. M. Thomas, E. Zeiter, N. Kosugi and H. Kuroda, J. Phys.: Condens. Matter, 1, 797-812 (1989).

37) M. Okada, P. Jin, Y. Yamada, M. Tazawa and K. Yoshimura, Surf. Sci., 566-568, 1030-1034 (2004).

38) H. Tang, K. Prasad, R. Sanjines, P. E. Schmid and F. Levy, J. Appl. Phys., 75, 2042-2047 (1994).

39) N. Kumar, K. Pourrezaei, B. Lee and E. C. Douglas, Thin Solid Films, 164, 417-428 (1988).

40) M. Mandl, H. Hoffmann and P. Kucher, J. Appl. Phys. 68, 2127-2132 (1990).

41) D. S. Shang, Q. Wang, L. D. Chen, R. Dong, X. M. Li and W. Q. Zhang, Phys. Rev. B, 73, 245427 (2006).

42) R. Dong, Q. Wang, L. D. Chen, D. S. Shang, T. L. Chen, X. M. Li and Q. Zhang, Appl. Phys. Lett., 86, 172107 (2005).

43) D. S. Shang, L. D. Chen, Q. Wang, W. Q. Zhang, Z. H. Wu and X. M. Li, Appl. Phys. Lett., 89, 172102 (2006).

44) R. Dong, Q. Wang, L. Chen, T. Chen and X. Li, Appl. Phys. $A, 80,13-16$ (2005).

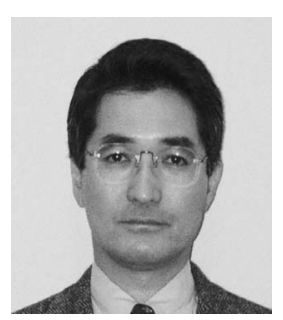

Masayuki Fujimoto is a professor of Innovative Joint Research Center and Graduate School of Science and Technology of Shizuoka University. He graduated from Science University of Tokyo in 1979 and the doctoral degree from University of Tokyo in 1988. He joined Taiyo Yuden Co., Ltd., Japan in 1979, and assigned to a general manager of the central research laboratories from 1989. He researched and developed various ceramic chip components and modules for advanced electric consumer devices. He received $19^{\text {th }}$ Fulrath Pacific Award the American Ceramic Society in 1996, and Technological Achievement Award of Society of Powder and Metallurgy Japan in 2002. He left Taiyo Yuden and joined Shizuoka University in 2004. His recent research field is related to nanoelelctronic ceramics. 